\title{
From Knowledge Engineering for Development to Development Informatics
}

\author{
Stefan Schlobach ${ }^{1}$, Victor de Boer ${ }^{1}$, Christophe Guéret ${ }^{2}$, Stéphane Boyera ${ }^{3}$ \\ and Philippe Cudré-Mauroux ${ }^{4}$ \\ 1 VU University Amsterdam, the Netherlands \\ k.s.schlobach@vu.nl, v.de.boer@vu.nl \\ 2 Data Archiving and Networking Services, Amsterdam, the Netherlands \\ christophe.gueret@dans.knaw.nl \\ 3 SBC4D consultancy, Toulouse, France \\ stephane@sbc4d.com \\ 4 eXascale Infolab, University of Fribourg, Switzerland \\ phil@exascale.info
}

\begin{abstract}
Knowledge Sharing is a key enabler of development of the rural poor. ICTs can play a critical role, providing for instance market data or weather information to sustenance farmers, or education to children in remote areas. While advanced knowledge technology has proven its use in many applications in the so-called developed world most of the tools cannot be easily applied in developing countries, because of restricted infrastructure, unsuitable modes of communication or ignorance of the local context. In the K4D tutorial at EKAW 2014 we argued that a new of kind of research in Knowledge Engineering is needed in order to make knowledge technology useful outside privileged developed countries. This research will have to include existing social and economic structures as fundamental requirements in order to be successful. Finally, we claim that this holds for a broader spectrum of subdisciplines of Computer Science, and not just for Knowledge Engineering, which lets us advocate Development Informatics: a joint forum for CS researchers who try to make their research relevant for the developing world as well.
\end{abstract}

\section{Introduction}

The tutorial "K4D - Managing and sharing knowledge in the developing part of the world" ${ }^{5}$ took place on November 24, 2014 as part of the workshop and tutorial program of the EKAW 2014 conference. It was targeted at the broader audience of Computer Science researchers interested in knowledge engineering and management and attracted an audience that was as mixed in provenance as in its professional interests.

Throughout this paper we will use the term knowledge sharing for the exchange of complex information (often contextual and not necessarily formalised). The most common forms of knowledge sharing do not rely on technology and are

\footnotetext{
${ }^{5}$ http://worldwidesemanticweb.org/events/k4d-2014/
} 
mostly oral or textual in natural language. Knowledge Technology on the other hand deals with the tools to use technology (e.g. computers, mobiles, radios, etc) to support knowledge sharing.

The tutorial was organized as a series of lectures, divided into four sessions:

1. Knowledge sharing in the development context

2. Knowledge technology in development practice

3. Knowledge technology as an enabler for development?

4. Development Informatics

The goal of the tutorial was to shed light on the specific conditions that Billion of people in developing countries face daily and that are too often unknown to the research community working on the design of knowledge information systems. This fell directly under the "diversity" topic of the conference in 2014 and more generally under the knowledge management theme of EKAW. The tutorial argued that the design of information systems for developing countries calls for specific tools, practices and patterns.

The goal was to equip the participants with an analytic toolkit to identify, in their own work, opportunities to broaden their research to be relevant to the people on the other side of the digital divide, as well as interesting research challenges that are specific to a development context.

There is a need for Computer Science to open up to development challenges. In this paper we argue for the emergence of a coherent CS sub-discipline, encompassing specific knowledge representation, information retrieval and software engineering research topics.

\section{Knowledge sharing in the development context}

Knowledge sharing can play an important role in overcoming poverty in the developing world. One of the most impressive, and famous, examples of the power of knowledge and knowledge sharing in the developing world is the work of Yacouba Sawadogo, a farmer from Burkina Faso, who extended existing knowledge in sustainable land management, which had an astonishing effect on efforts to re-green the Sahel zone. The power of his approach was the local context in which this knowledge was created and embedded as well as the spread of the knowledge by means of the almost missionary power of the knowledge sharing capabilities of Yacouba and others involved. ${ }^{6}$

By making knowledge sharing more effective and scalable, Knowledge Technology can become a key enabler of development for the rural poor. Before analysing the specific requirements to Knowledge Technology in a developing context we report on previous research into knowledge sharing case studies in rural, developing regions. For a number of cases, we have already developed and deployed ICTs using a living-labs method [1]. Other cases are currently under development.

\footnotetext{
${ }^{6}$ There is an documentary made about Yacouba Sawadogo work: http://www. 1080films.co.uk/project-mwsd.htm.
} 
Market Information Sharing. This use case was developed in a livinglabs collaboration between Malian sustenance farmers, radio hosts, a local NGO and CS4D researchers [1]. Local farmers expressed the need to share information about prices and availability of local produce such as honey or cereals. A Market Information System (MIS) called RadioMarché was developed in collaboration with the stakeholders. RadioMarché allows for information creation and access using simple mobile phones through voice interfaces in local languages. The market information is locally relevant for the farmers and their clients, but also monitored on an aggregate level by the local NGO.

Radio Information Platform. A second case and system was developed in the same area. The Furoba Blon system allows radio hosts to collect information from remote villages using citizen journalists who call in with their news items. This is a more generic platform in the sense that the type of information is less restricted. Local radio hosts manage and access the information using a webinterface or a voice-interface using their mobile phones in a local language [1].

Weather prediction using Pluviometric data. Weather prediction is important for planning, securing and assessing farming and its products. Global weather prediction systems exist, but due to climate change and local conditions, it is important to use local observation data to analyse data on local levels. In rural Burkina Faso and Mali, we are developing a system for gathering, sharing and aggregating pluviometric data. Local farmers expressed the need for effective and efficient creation, curation and sharing of rainfall and weather data. This data is locally shared and can be combined with (Open) satellite data into a larger observation system. Based on aggregate knowledge, one can understand large scale hydrologic processes, such as flooding, irrigation and vaporization.

Land rights. More complex knowledge is being created and shared in this case in rural Burkina Faso which centers around land rights. Local farmers are often unaware of the complex issues around land ownership and usage rights. This is sometimes exploited by others to illegally use land for mining or building purposes. Local NGOs have mapped these land rights and seek solutions to share this knowledge amongst farmers in an effective and timely manner.

Innovative Farming Techniques. In collaboration with local NGOs, innovative farmers in rural West-Africa are developing innovative farming techniques to deal with soil degradation and desertification. Examples of these techniques are the use of stone walls or the timely digging of holes (so called zaï) to recover and retain water on farmland. This complex and locally created knowledge is currently being aggregated by the NGOs to be shared between farmers using folders, videos and extension workers. Here, knowledge representation and knowledge sharing techniques can assist in increasing the reach of these techniques.

Education statistics. Among the different approaches for improving education in deprived part of the world, the foundation One Laptop Per Child (OLPC) decided to go for giving one laptop to every child and let them progress on their own under the guidance of a teacher. Monitoring this learning process is needed as educators and state institutions need to know how all the young learners are 
performing. Several solutions have been proposed to gather and aggregate this data [2], turning private nominative data into anonymous public statistics.

These case studies each bring their own specific challenges to using ICT and knowledge technologies. However, we can make a number of generalizations about the specific challenges and approaches to knowledge technologies for development.

\section{Technology for Knowledge Sharing in Development}

Common to all these examples is that there is a clearly identified need for knowledge sharing, and that there is some basic infrastructure to apply Knowledge Technology. On the other hand, in none of the examples can state-of-the-art Knowledge Technology directly be applied. In the following sections we will describe some initial attempts to close this gap, and explain why this is non-trivial, both from a technological, methodological and socioeconomic perspective.

\subsection{Requirements and challenges}

First of all, any knowledge technologies need to be embedded in existing infrastructure and social context to ensure local uptake and sustainability of solutions. This calls for "downscaling" of knowledge technologies solutions to existing infrastructures [3]. These include mobile telephony as main intercommunication device. Where in rural developing areas personal computers and tablets are hardly used, mobile devices have been recognized as having the greatest potential for development [4]. In Africa, mobile telephony has become the primary mode of telecommunication [5]. More specifically, most mobile phones in use in these areas are simple $(2 \mathrm{G})$ compared to current generation smartphones or feature phones. To ensure the maximum reach of knowledge technologies, we therefore should consider making them available through voice or text messaging.

Another information sharing platform that is currently used in large parts of the developing world is that of radio. Radio is for example still the most popular mass-medium in Africa and currently used to distribute a wide variety of information amongst listeners. Examples include services for farmers (market prices or weather reports) or health services. Incorporating radio as a platform for Knowledge Technologies can drastically improve its reach [6].

It is also paramount that ICT solutions -including knowledge technologies, operate within existing socioeconomic and business processes. To ensure that developed technologies are sustainable, they need to be owned by local stakeholders. At the same time, the socioeconomic context needs to be understood to a degree where the Knowledge Technology is able to be used by the users it is designed for. For example, if local power structures prohibit users to enter information in a system, such a system will lose effectiveness. 


\subsection{Example for paradigm shifts}

There are many challenges and opportunities for new approaches to knowledge technologies for development. This requires an open mind and in some cases a radical shift in paradigms. For example, where most knowledge technologies assume broadband networks to transfer information, in low-connected environments this does not hold. In these cases, information can be distributed using the mobile network or through radio. Sneakernets ${ }^{7}$ employ physical transportation of data-carrying devices, which in some cases can result in high bandwith throughput (albeit with a high latency). For existing knowledge technologies we can consider to what extent they can use these alternative networks and as computer scientists, we can adapt them where necessary. We present two examples of current work on using Linked Data technologies in these new paradigms.

- Linked Data and Voice Technologies. In [7], the authors present work on using Voice access for simple mobile phones to Linked Data for to rural communities in the Sahel region. This work builds on RadioMarché, a market information system (MIS) which can be accessed using first-generation mobile phones. Linking the locally produced market data to other data sources will increase its value for use by local NGOs but also for subsistence farmers themselves. The RadioMarché data was made available as Linked Open Data. At the same time, the authors present an ontology for describing RDF resources as voice recordings in multiple languages. These voice fragments can then be used in a voice interface that can be accessed by simple mobile phones. The authors present a prototype demonstrator that provides voicebased access to this linked market data. As such, these are first steps towards opening Linked Data to local users that do not have appropriate hardware to produce and consume Linked Data.

- Entity Registry System Semantic Web solutions typically assume the availability of Web architectures: including access to global network access. However, in rural environment, these assumptions do not hold. In many cases where internet connectivity is available, these are not stable. The Entity Registry System (ERS) [8] is software that provides Linked Data solutions for local "not-always-online" networks. ERS is a decentralised registry which is designed to be read/write, track the provenance of the contributions and be usable offline and online. This registry creates a global data space where every user is able to say something about any entity. Just like Wikis allows for the collaborative of a web site, ERS will enable the collaborative editing of a registry on a de-centralised basis. The data associated to an entity is its description, recorded as RDF data. An implementation of ERS was developed that allows for effective information exchange for the Sugar Learning Platform ${ }^{8}$ originally developed for the XO laptop of the One Laptop Per Child initiative (OLPC) ${ }^{9}$.

\footnotetext{
${ }^{7}$ http://en.wikipedia.org/wiki/Sneakernet

8 http://sugarlabs.org/

9 http://one. laptop.org/
} 
- Localising Open Data The "Open" movement also has caught on in International Development and a number of development researchers and practitioners recognize the benefits of building openness into policies and technologies for development. As is noted by Davies and Edwards [9]: "Governments, companies and non-governmental organisations (NGOs) across the world are increasingly exploring how the publication and use of open and linked data can have impacts on governance, economic growth and the delivery of services". However, it remains a challenge to bridge the digital divide and have open development data have significant impact for the rural poor themselves since these people often lack access to data portals on the Web or lack the literacy to interpret the data. The World Bank has recently experimented with bringing Open Data about development contracting to rural villages in Indonesia and Kenya ${ }^{10}$. They developed print material that was shared with local community members in intensive interactive sessions. This is an example of sharing knowledge using alternative modalities. Other examples are the usasge of icon-based communication or development of video or animation material to share knowledge.

\section{Knowledge Technology as an enabler for development?}

In the previous sections we have provided examples of potentially useful and promising applications of Knowledge Technology in development. An important basis for these approaches was the local embedding and the careful adjustment to the social context of each individual application of Knowledge Technology.

In this section we want to expand on this theme, by discussing Knowledge Technology in development in a broader socioeconomic perspective. First, in Section 4.1 we will briefly revisit existing arguments with respect to ICT4D in general, before identifying some specific challenges to Knowledge Technology in Section 4.2.

\subsection{Knowledge Technology in ICT4D}

The application of information and communication technologies (ICT) in development might be considered at first thought to be an uncontroversial tool for development, but there have been heavy discussions about their role and impact from sociological, political and economic perspectives. In this section we will briefly summarise some of these arguments to clarify the challenges and responsibility researchers in Knowledge Technology face when working in development case studies.

What is Development? Originating in the ideas of the Enlightenment, development is about economic, social and political progress, and technology seen as one of the driving vehicle for this kind of progress. This is a disputed, and

$\overline{10}$ http://www . open-contracting.org/bringing_open_contracting_data_offline 
highly European, notion, where progress is often considered an essential part of the functioning of our societies. It should, however, be noted that this a very biased notion, which is meaningless, and non-desirable, in many societies. This undermines the very foundations of many approaches to ICT4D, which aims at transforming societies to resemble what we consider to be successful role-models. This colonial view is very often at the core of misunderstanding in ICT4D.

Another argument questions the role of ICT for benefitting society: competition about technological leadership can lead to innovation, but mostly for the those already in a leading position, which increases, rather than decreases, global disparity. In words of Tim Unwin, an outspoken critics of traditional ICT4D approaches, "technology has all too often been used mainly to enable the rich and privileged to retain their positions of economic, social and political power" [10].

Neo-liberal approaches have mostly focussed on trade rather than aid, free markets and privatisation of state-owned companies. As a consequence $70 \%$ of Ghana Telecom is, e.g., now owned by Vodafone. Although some of this kind of involvements potentially increases the availability of advanced technology critics identify the risks that this technology is not used in the interest of the people, but mostly to increase the gain of usually Western companies.

What is the role of information and communication technologies for development? In this paper we adopt a broad view ICT, meaning all kinds of electronic means of capturing, processing, storing and communicating information, such as computers, phones, Internet, radio or tv. Access to information has been identified as one of the core human rights, and Universal Declaration on Human Rights by the United Nations in 1948 states in Article 19: "Everyone has the right [...] to seek, impart information and ideas through any media and regardless of frontiers." In this respect there is nowadays a central role for ICTs, as they are one of the central carriers of information in modern times. Considering this role of ICTs for information and knowledge sharing, access to and usability of such technology is of crucial importance.

The potential benefits of ICTs in development are therefore enormous: at the most abstract level Knowledge Technology can play an important role in knowledge and information sharing, with potential contributions to empowerment, education, advances of democratic process and emancipation. Given the previous discussion about ICT4D, though, this optimistic look has to be relativized, as those positive benefits are not guaranteed, and require a careful analysis of the application scenario at hand. Some of the risks have been identified in the ongoing debate about ICT4D.

The big debate about ICT4D The debate about ICT4D has been ongoing for decades. From an economic perspective the importance of science and technology for economic growth has been emphasised often referring to positive examples from Asia, where a combination of skilled labour forces and excellent infrastructure turned ICTs into main vehicles for impressive economic growth. ICTs in these cases helped accessing information and facilitate trade, which ensured 
those countries a strong position on growing marked. Many questions remain though in how far those effects can be reproduced more globally. On the African continent, e.g., there is hardly any production of hardware or software, not is there a sufficiently educated labour-force to deliver ICT services even to the own people. This means that the supply chains of ICT remain between US, Europe, India, China, Taiwan, South Korea, Japan, Russia etc., with Africa paying for services without further profiting economically.

Many critics have pointed to the environmental impact of current approaches to ICT4D where hardware, sometimes old leftover hardware from developed countries, is delivered to the developing countries. Combine this with a lack of expertise to apply this hardware in local contexts, development aid of ICTs often simple results in uncontrolled dumping of old, and often highly toxic, technology.

From a social-cultural and socioeconomic viewpoint new, Western, ICT approaches are often radically deviating from traditional communication models, with two possible unwanted outcomes: either the technology is simply not used and the development effort wasted at best, or the technology destroys existing and successful communication structures, replacing them with often unsuitable and untested new paradigms.

\subsection{ICT4D Specific Challenges to KT}

The case studies described in Section 2 have shown us that users in poor rural areas are as we speak creating knowledge in the field, in schools, etc. The stakeholders express the need for more effective and efficient creation, curation and sharing of this knowledge. Currently, they are hindered in doing so using ICTs mainly because of the lack of infrastructure (Internet access, lack of electrical power, etc.); incompatible interfaces (which do not take into account local languages or literacy levels) and the lack of generic platforms for creating bottom-up knowledge sharing solutions. This calls for new designs of knowledge sharing architectures that take into account these issues. Our ongoing efforts helped shape three distinct aspects to focus research agendas on:

Interfaces Such architectures should allow knowledge creation, curation and sharing through relevant interfaces that take into account local languages and levels of literacy. Voice-based [1] or icon-based interfaces have shown great promise here, especially when combined in multi-modal interfaces.

Infrastructure Similarly, the systems should be designed to work with nonstandard infrastructures. On the one hand, there is often a lack of persistent power grid, internet connectivity and ownership of computing devices. On the other hand, mobile networks and simple first- or second-generation mobile phones are widespread in many developing areas. Effective knowledge sharing platforms should make use of these assets. At the same time, small hardware solutions are affordable both in price and cost of ownership (low power consumption). They are robust solutions to serve as data sharing nodes [3]. 
Contextualisation of knowledge A characteristic of these cases is that often, the information that is created is primarily locally used. However, a secondary usages lies in the aggregation to higher levels (regionally, countrylevel, global). The other way around, this more generic data is also relevant locally when combined with the local information. Knowledge sharing platforms should take this issue into account. For example, when global (internet) connectivity is temporarily unavailable, knowledge can still be shared locally using local networks. In Table 1, we list the use cases and some characteristics of the knowledge being shared. This includes where it is created, uses of the knowledge (primary and secondary) and whether Peer-to-Peer exchanges are needed. In some use cases, the knowledge being exchanged is fairly simple. In other cases, the knowledge is much more complex. The innovative farming techniques are made up of relatively complex sequences of farming actions depending on weather, soil structure etc. Table 1 shows the diversity in the complexity of the knowledge across the use cases. The complexity will have its consequences for the interface design as well as how knowledge is shared and aggregated. A generic platform should be able to deal with the creation, sharing and curation of knowledge of different levels of complexity.

\begin{tabular}{|c|c|c|c|c|}
\hline Use case & Knowledge creation & usage & P2P exchanges & Complexity \\
\hline Market Information & local & local+global & important & simple \\
Radio Platform & local & local & not needed & simple \\
Pluviometry & local+global & local & not needed & simple \\
Land rights & local+global & local & useful & complex \\
Farming techniques & local+global & local & useful & complex \\
Education statistics & local & global & not needed & simple \\
\hline
\end{tabular}

Table 1. Overview of the different aspects of the knowledge in the use cases. Knowledge creation and usage : the importance of locally created knowledge (e.g. produced by the end users) versus its global counter part (e.g. governmental or country level). P2P exchanges : amount of local communication among peers from a different level of communications. Complexity : appreciation of the number of entities and level of communications that can be observed, leading to simple to more complex systems.

Our experience so far through different projects ${ }^{11}$ taught us that it is important not only to consider all these specific topics together in one holistic view but also to ensure the target population and ICT experts are always part of the discussions. We consider this as part of the bottom-up living-labs approach,

11 See the papers cited in this paper but also the community landing pages http: //worldwidesemanticweb.org/ and http://w4ra.org/ for an overview. 
working from local needs and focus on markets and using locally sustainable ICTs.

\section{From KT4D to Development Informatics}

There is a duality in Computer Science as it can be considered either as a discipline or as a tool. As a tool computer science is key to processing large amount of data whereas the development of the novel algorithm needed for such computation would appeal more to the discipline facet of Computer Science. The consequence of doing ICT4D without Computer Scientists is that standard and simple ICT solutions are applied (and often fail) following a "one size fits all" idea. The expertise needed to adapt existing technical solutions and develop new solutions when those existing can not fit is missing. Multi-disciplinary approaches combining the expertise of ICT4D practitioners and that of Computer Scientists hold the promises of better optimised computational systems.

The reasons this is only rarely happening now have not be sought in the lack of interest for Computer Scientists to help improve the life of underprivileged world citizens, nor in the lack of motivation for ICT4D experts to collaborate with Computer Scientists. There are fundamental thresholds Computer Scientists have to overcome:

- There is an intrinsic difficulty to work in multi-disciplinary contexts. As we have argued, successful development and deployment of Knowledge Technologies for development will have to be done in collaboration with experts able to investigate the socioeconomic context. Other collaborations will include language technology specialists or hardware designers.

- There is a very high threshold to work in ICT4D:

- The knowledge gap is huge (on both sides). On the one hand, Computer Scientists do not know about development issues, and on the other hand, ICT4D practitioners and development experts are unaware of the benefits that CS research can bring.

- Intrinsic uncertainty and carefulness of researchers to work in new research fields.

- Scientific recognition (still) rather limited. To be able to draw consistent and sustained support contributions from Computer Scientists, this recognition will have to grow larger.

- The scientific challenges of Computer Science in a development context are not well understood

- In most cases there is a physical distance between the researchers and the target population. This causes development challenges to remain unknown to researchers and makes working efficiently difficult.

- There is also a cultural distance between researchers and target population, which, together with language barriers cause for difficulties in communication. 
- It can finally be noted that, in an ever going globalisation of the World economy where competition among (groups of) countries is tough levering funding from on part of the World to help another can prove to be challenging.

Besides the necessary discussions among all those are concerned ${ }^{12}$, we believe the definition of a coherent research discipline is a first step into solving them. We therefore propose establishing Development Informatics as a sub-discipline of Computer Science. Development Informatics research integrates development specific problems into computer science research efforts. These can be in Distributed Systems, Computational Linguistics, Human-Computer Interaction, or, in the case of K4D: Knowledge Engineering. Those Computer Science experts will face common development-related challenges: technologically, as well as legally, ethically, sociologically and politically.

In this Development Informatics, some not so common research questions can emerge related to the specific challenges listed above. For example, looking at the specific topics of knowledge representation, information retrieval and software engineering :

\section{- Knowledge representation}

- How to relate textual and vocal data representations to enable voice interfaces to data?

- What level of common semantics can be reached among several communities speaking all a different dialect among them ?

- What are the impact of the digitization of content and data collection on the life of people living in developing economies?

- Information retrieval

- Can visualisation and voice be leveraged to bridge the literacy gap in computer-human interfaces?

- What is the role of information sharing in development ${ }^{13}$ ?

- Can insights from development experts be used to better understand the dynamics of informal economies and drive the design of optimised computational systems?

- Software engineering

- What are the sensible assumptions to make when designing a computational system for a developing economy?

- What are the Technological Challenges for system change?

- Can data intensive computational systems be made to fit contexts with limited resources (hardware, connectivity, ...) ?

$\overline{12}$ Face-to-face as during this tutorial and other events we organise but also on-line in discussion community groups.

${ }^{13}$ Eventually focusing on development related datasets such as health-care data, aid money spending, votes, contracts, or education indicators. 


\section{Conclusion}

Supporting knowledge sharing through state-of-the-art knowledge technology in the developing world can not be achieved by simply deploying well-established ICT solutions in a top-down manner. The Knowledge Engineering community has to take on the problem of development by increasing the applicability of its technologies in developing countries. For this, the global discussion of ICT4D has to be understood, with its implications on global and local political, socioeconomic, and socio-cultural processes but, most and foremost, the local context of the applications has to be understood, which often implies a radical paradigm shift. In previous work we identified 3 main dimensions in which the knowledge engineering community has to broaden its approach: study alternative (often weaker) infrastructures, novel interface paradigms, and contextualisation. In this paper, we claim that these findings call for an even larger effort, the creation of a new subdiscipline of Computer Science, which we call Development Informatics.

\section{References}

1. Gyan, N.B., de Boer, V., Bon, A., van Aart, C., Akkermans, H., Boyera, S., Froumentin, M., Grewal, A., Allen, M.: Voice-based web access in rural africa. In: WebSci, ACM (2013) 122-131

2. Verma, S.: The quest for data. http://www.olpcsf.org/node/204 (2014)

3. Guéret, C., de Boer, V., Schlobach, S.: Let's downscale linked data. Internet Computing, IEEE 18 (2014) 70-73

4. Boyera, S.: Mobile web for social development (MW4D). Technical report, W3C (2008) http://www.w3.org/2008/MW4D/, accessed 19th March 2010.

5. UNCTAD Secretariat: Science and technology for development: the new paradigm of ict. In United Nations Conference on Trade and Development, ed.: Information Economy Report 2007-2008, United Nations Publication (2007) http://unctad.org/en/Docs/sdteecb20071_en.pdf, accessed 8th August 2011.

6. Bon, A., Boer, V.D., Leenheer, P.D., Aart, C.V., Baah, N., Froumentin, M., Boyera, S., Allen, M., Akkermans, H.: The web of radios- introducing african community radio as an interface to the web of data. In: Proceedings of the 1st International Workshop on Downscaling the Semantic Web - Downscale2012. ceur-ws.org/vol844. (2012)

7. de Boer, V., Gyan, N.B., Bon, A., Tuyp, W., van Aart, C., Akkermans, H.: A dialogue with linked data: Voice-based access to market data in the sahel. Semantic Web (2013)

8. Guéret, C., Cudré-Mauroux, P.: The entity registry system: Publishing and consuming linked data in poorly connected environments. ERCIM News 2014 (2014)

9. Davies, T., Edwards, D.: Emerging implications of open and linked data for knowledge sharing in development. IDS Bulletin 43 (5) 117-127 (2012)

10. Unwin, T.: ICT4D. Cambridge University Press (2010) 